\title{
Pre-Printed Versus Handwritten Post-It Notes' Impact on Survey Response in Hard to Recruit Survey Recipients: A Randomized Controlled Trial in Veterans with PTSD
}

\section{Disability}

\author{
Maureen Murdoch (Maureen.Murdoch@va.gov) a, b, c \\ Michele Spoont (Michele.Spoont@va.gov) ${ }^{b, c}, \mathrm{~d}, \mathrm{e}, \mathrm{f}$ \\ Eileen Harwood (harwo002@umn.edu) $)^{\text {bg }}$ \\ Barbara Clothier (Barbara.Clothier@va.gov) ${ }^{b}$ \\ Ann Bangerter (Ann.Bangerter@va.gov) ${ }^{b}$ \\ Shannon Kehle-Forbes (Shannon.Kehle-Forbes@va.gov) b, c \\ Nina Sayer (Nina.Sayer@va.gov $)^{b, c, f}$ \\ Melissa Partin (Melissa.Partin@va.gov) ${ }^{\text {b,c }}$
}

${ }^{\text {a }}$ Section of General Internal Medicine

${ }^{\mathrm{b}}$ Center for Chronic Disease Outcomes Research, Minneapolis VA Health Care System

${ }^{c}$ Department of Internal Medicine, University of Minnesota Medical School

${ }^{\mathrm{d}}$ National Centers for PTSD, Pacific Islands Division, Department of Veterans Affairs

${ }^{\mathrm{e}}$ Department of Psychiatry, University of Minnesota Medical School

${ }^{\mathrm{f}}$ Department of Psychology, College of Liberal Arts, University of Minnesota

g Division of Epidemiology and Community Health, University of Minnesota School of Public Health

\section{Corresponding author:}

Maureen Murdoch, MD, MPH

Center for Chronic Disease Outcomes Research (111-0)

Minneapolis VA Medical Center

One Veterans Drive

Minneapolis, MN 55417

Acknowledgments: This work was supported by the VA Health Services Research and

Development Service (IIR-09-359, CDA-09-020, CIN-13-406). We thank Siamak Noorbaloochi for his input. 


\begin{abstract}
Our goal was to see if affixing pre-printed Post-it note messages to a mailed survey would generate response rates similar to when handwritten Post-it notes were used in hard to recruit survey recipients. The study was a nested, randomized controlled trial of 575 individuals from a cohort of 2,100 United States Veterans with PTSD disability. Analysis was intention-to-treat. Survey response rate was $38.5 \%$ in the pre-printed Post-it note arm and $44.9 \%$ in the handwritten Post-it note arm $(p=0.09)$. In non-inferiority testing, pre-printed notes' response rate exceeded the $10 \%$ margin of inferiority but was less than the $15 \%$ margin when compared to handwritten notes.
\end{abstract}

Tags: Randomized Trial, Patient Surveys, Response Rate, Post-it Notes 


\section{Introduction}

In university samples, affixing handwritten Post-it notes to inter-office surveys has doubled response rates; they seemed particularly useful in improving response rates to lengthy, 24-page surveys (Garner, 2005). Garner (2005) speculated that handwritten Post-it messages triggered favorable reciprocal responses among his survey recipients, because they viewed his requests to complete and return the survey as a personal favor. Garner's (2005) handwritten Post-it notes may also have increased the perceived personalization of his surveys. Meta-analyses have consistently shown that personalized mailings, such as saluting survey recipients by name or closing cover letters with the principal investigator's handwritten signature, improves survey response rates compared to less personalized methods (Edwards et al., 2009).

In other settings, the impact of handwritten Post-it notes on survey response rates has been more modest, yielding at most a 3.5\% increase in response rates (Cowie, Morgan, \& Gulliford, 2011; Gendall \& Healey, 2008). Since Post-it notes are inexpensive, currently costing less than one US penny per note, even this smaller boost may be worthwhile. The labor required to handwrite the notes, on the other hand, can be substantial— particularly for very large studies. Our goal, therefore, was to see if pre-printed Post-it notes would generate response rates similar to those seen for handwritten Post-it notes. Because of the increased personalization associated with handwritten Post-its, we anticipated that handwritten Post-it notes would generate higher response rates than would pre-printed Post-it notes. However, pre-printed Post-its represent substantial time and labor savings that could prove desirable as long as the response rates obtained were not grossly inferior to those obtained with handwritten Post-its. In addition to traditional superiority testing, therefore, we also examined the non-inferiority of pre-printed Post-its notes to handwritten Post-its. 


\section{Methods}

The study is a randomized controlled trial nested in a follow up survey of 2,100 United States Veterans. The Veterans were cohort members who had applied for Department of Veterans Affairs posttraumatic stress disorder (PTSD) disability benefits between 1994 and 1998 and had completed 2 prior mailed surveys in 1998 and in 2006 (Murdoch et al. 2017). The Minneapolis Veterans Affairs Medical Center's Subcommittee for Human Studies approved the protocol. Veterans consented to participate in the present study by returning completed surveys. Data were collected between May 1, 2011 and November 1, 2012.

We followed a modified Dillman (2007) approach to recruitment. All 2,100 eligible Veterans received a pre-notification letter about the coming survey. One week later, they received a cover letter, 20-page questionnaire that asked about PTSD symptoms and functioning, and \$10 cash incentive (Request \#1). The cover letter invited Veterans to complete and return the survey, described the risks and benefits of participation, and informed them that their participation was completely voluntary. At two-week intervals non-respondents received a post-card reminder (Request \#2) and second mailing of the survey (Request \#3). After the third request there were 1,421 survey respondents ("early respondents”) and 14 deceased Veterans, 36 invalid addresses, 53 refusals, and 1 Veteran misclassified as an early respondent. The remaining 575 survey recipients were classified as "hard to recruit." Using computer-generated, simple random allocation without replacement, "hard to recruit" Veterans were randomized to receive a handwritten or pre-printed Post-it note attached to a final, mailed survey (Request \#4). Post-its for both conditions were star-shaped and yellow with identical text: "Thank you for completing the survey. We really appreciate it!" Post-its were affixed to the survey cover's upper right-hand corner. We opted to use Post-it notes at this final stage of recruitment because we believed that 
the increased personalization and triggers for reciprocal responses they offered would be most useful when trying to elicit cooperation from harder to recruit individuals.

Table1 shows the overall sample's sociodemographic characteristics, as well as those of early respondents and hard to recruit survey recipients by randomization group. On net, early respondents were approximately 2 years older than the hard to recruit survey recipients.

Otherwise, hard to recruit survey recipients did not differ importantly or statistically significantly from the early respondents. The characteristics of hard to recruit survey recipients randomized to receive handwritten Post-it notes did not differ importantly or statistically significantly from those randomized to receive pre-printed Post-it notes.

Analysis was intention-to-treat. For the outcome, we used the American Association for Public Opinion Research's (2015) response rate definition number 1 (response rate = the number of hard to recruit survey recipients who returned a survey in each study arm divided by the total number of hard to recruit recipients randomized to that arm). Analysis proceeded in two stages. In the first, we conducted a traditional two-tailed superiority test to see if response rates obtained with one method were statistically significantly higher than with the other method. Although we anticipated response rates would be higher in the handwritten arm than in the pre-printed arm, the two methods had never been compared to our knowledge. It was also possible that preprinted Post-its would prove superior to handwritten ones. Were pre-printed Post-its to prove superior, there would be no need to proceed to non-inferiority testing. We set our two-tailed alpha to 0.05 and used a conventional $\mathrm{p}$-value $\leq 0.05$ to denote statistical significance for this analysis.

In the second stage, we tested for the non-inferiority of pre-printed Post-its relative to handwritten Post-its. We specified two non-inferiority margins: 10\% and 15\%. Were response 
rates to pre-printed Post-its to be $10 \%$ lower than handwritten Post-its or less, we believed that many researchers would choose them over handwritten Post-its because of their convenience. Were response rates to pre-printed Post-its to be more than $15 \%$ lower than handwritten Post-its, however, we suspected most researchers would choose handwritten Post-its. As with the primary analysis, alpha was set to 0.05 .

For our non-inferiority analysis, the null hypothesis was:

$$
\left.H_{0}: \text { Response rate } \text { preprinted }_{-} \text {Response rate } \text { Randwritten } \leq-10 \% \text { (or } \leq-15 \%\right)
$$

The alternative hypothesis was:

$$
\left.H_{1} \text { : Response rate } \text { preprinted }_{-} \text {Response } \text { rate }_{\text {handwritten }}>-10 \% \text { (or }>-15 \%\right)
$$

We calculated non-inferiority $z$ scores following Laster, Johnson, \& Kotler (2006). Noninferiority is accepted -that is, the null hypothesis is rejected-if the calculated $z$ score is greater than 1.645 .1 .645 is the $95^{\text {th }}$ (1-alpha) percentile of a standard normal distribution. We used IBM SPSS Statistics (version 19) statistical packages for analyses.

\section{Results}

$111(38.5 \%)$ Veterans returned surveys in the pre-printed Post-it arm and 129 (44.9\%), in the handwritten Post-it arm. Under traditional superiority testing, this difference of $-6.4 \%$ was not statistically significant (two-tailed $95 \% \mathrm{CI}$ around the difference $=1.7 \%$ to $-14.3 \%, \mathrm{z}=1.68, p=$ 0.09; lower bound, one-tailed $95 \% \mathrm{CI}=-13.2 \%$ ). The calculated $z$ score for $10 \%$ non-inferiority was $0.88(p=0.19)$; for $15 \%$ non-inferiority, the $z$ score was $2.10(p=0.02)$. As can be seen from Table2, the characteristics of respondents to surveys with handwritten Post-it notes did not differ significantly from that of respondents to surveys with pre-printed Post-it notes. Further, their characteristics did not differ significantly from the hard-to-recruit non-responders. 


\section{Discussion}

In this study, handwritten Post-it notes generated a slightly higher response rate than did preprinted Post-it notes, but the difference was not statistically significant using traditional testing for superiority. In non-inferiority testing, pre-printed notes' response rate exceeded the $10 \%$ margin of noninferiority but not the $15 \%$ margin when compared to handwritten notes. Respondents' sociodemographic characteristics did not differ by the type of Post-it note they received. Thus, our data do not identify a clear choice for which Post-it note researchers might use. For some, a possible response rate improvement of $10 \%$ might be worth the time and effort required to produce handwritten notes; others might prefer pre-printed notes' convenience.

To our knowledge, only Garner (2005) has obtained large boosts in response rates using handwritten Post-it notes compared to controls (e.g., increases of up to 40 full percentage points). His studies all involved surveys asking about University climate sent to University stakeholders through interoffice mail. Post-it notes are possibly more effective in such settings. In representative national and city-wide studies in New Zealand, handwritten Post-it notes' net effect on mailed survey response rates have ranged from -0.8 to $3.5 \%$ (Gendall \& Healey, 2008). In a probability sample of inner city residents with historically low survey response rates, handwritten Post-it notes boosted response rates to a mailed survey about health care utilization by a statistically non-significant 3.3\% (Cowie et al., 2011).

Garner's (2005) original Post-it note message, "Please take a few minutes to complete this for us. Thank you!" was specifically designed to trigger favorable reciprocal responses among survey recipients. Gendall and Healey (2008) used the same message with less success in their New Zealand probability samples. However, unlike most researchers, Gendall and Healey (2008) affixed Post-it notes to their cover letters, not to the questionnaires. This may not have been 
optimal. For example, Garner (2005) believed that Post-it notes affixed to the actual questionnaire would elicit "greater attention to the material" (pg. 230). Other Post-it note messages have included "Many thanks for all your help" (Cowie et al., 2011), "Please complete and return this as soon as possible, [signature of researcher's first name]" (Tilbrook et al., 2015), and our own, "Thank you for completing the survey. We really appreciate it!" Some messages may be more motivating than others: this might be explored in future research.

Our yellow Post-it notes were also in the shape of a star, while other investigators have used yellow squares. Different note shapes could influence survey recipients' reactions to the messages therein. We reserved Post-it note prompts for our most difficult to recruit survey recipients, but most researchers have used them with their initial mailings (Cowie et al., 2011; Garner, 2005; Gendall \& Healey, 2008; Tilbrook et al., 2015). Holding Post-it notes in reserve certainly reduces the costs and time required to produce them, but it may be that they are more effectively used with earlier mailings. Because our sample and survey topic were highly specialized, findings may not generalize to other populations, to other survey topics, or to onetime, cross-sectional studies.

The use of Post-it notes in mailed surveys has been little studied, particularly pre-printed ones. Given their low costs, additional studies comparing pre-printed versus a variety of other conditions would help clarify their role in boosting response rates. Future research might also examine the effect of different messages, different note shapes, or different timing strategies (e.g., with the first or last mailed prompt). 


\section{References}

American Association for Public Opinion Research. 2015. Standard definitions: final dispositions of case codes and outcome rates for surveys (8th ed.). American Association for Public Opinion Research, Oakbrook Terrace, IL.

Cowie, L., Morgan, M., and Gulliford, M. 2011. Handwritten 'post-it' notes, questionnaire formats and response to a postal questionnaire survey. International Journal of Epidemiology 40(1): 254-255.

Dillman, D. 2007. Mail and internet surveys: the Tailored Design Method (2 ${ }^{\text {nd }}$ ed.). John Wiley \& Sons, Inc., Hoboken, NJ.

Edwards, P., Roberts, I., Clarke, M., DiGuiseppi, C., Pratap, S., Wentz, R., and Kwan, I. 2009. Methods to increase response to postal and electronic questionnaires. Cochrane Database of Systematic Reviews, Art. N. MR000008.DOI:10.1002/14651858.MR000008.pub4(3).

Garner, R. 2005. Post-it note persuasion: a sticky influence. Journal of Consumer Psychology 15(3): 230-237.

Gendall, P. and Healey, B. 2008. Alternatives to prepaid monetary incentives in mail surveys. International Journal of Public Opinion Research 20(4): 517-527.

Laster, L., Johnson, M., and Kotler, M. 2006. Non-inferiority trials: the "at least as good as" criterion with dichotomous data. Statistics in Medicine, 25(7), 1115-1130. doi: $10.1002 / \operatorname{sim} .2476$

Murdoch, M., Spoont, M., Kehle-Forbes, S., Harwood, E., Sayer, N., Clothier, B., and Bangerter, A. 2017. Persistent serious mental illness among former applicants for VA PTSD disability benefits and long-term outcomes: symptoms, functioning, and employment. Journal of Traumatic Stress 30(1): 36-44. 
Tilbrook, H., Becque, T., Buckley, H., MacPherson, H., Bailey, M., and Torgerson, D.J. 2015. Randomized trial within a trial of yellow 'post-it notes' did not improve questionnaire response rate among participants in a trial of treatments for neck pain. Journal of Evaluation in Clinical Practice 21(2): 202-204. 
Table 1. Sociodemographic Characteristics

\begin{tabular}{|c|c|c|c|c|}
\hline \multirow[t]{2}{*}{ Characteristic } & \multirow{2}{*}{$\begin{array}{l}\text { Overall } \\
N=2,100\end{array}$} & \multirow{2}{*}{$\begin{array}{c}\text { Early } \\
\text { Respondents } \\
\text { (responded } \\
\text { prior to } \\
\text { randomization) } \\
\\
n=1,421\end{array}$} & \multicolumn{2}{|c|}{$\begin{array}{l}\text { Randomized to a Post-It } \\
\text { Note Condition }\end{array}$} \\
\hline & & & $\begin{array}{c}\text { Handwritten } \\
n=287\end{array}$ & $\begin{array}{c}\text { Pre- } \\
\text { Printed } \\
n=288\end{array}$ \\
\hline Current age in years, mean (SD) & $56.9(9.5)$ & $57.3(9.1)^{* * *}$ & $54.9(10.5)$ & $55.7(9.8)$ \\
\hline \multicolumn{5}{|l|}{ Race, $\%$} \\
\hline White & 72.6 & 72.9 & 70.4 & 71.2 \\
\hline Black or African American & 17.7 & 17.8 & 17.8 & 19.1 \\
\hline Hispanic & 5.5 & 5.3 & 7.0 & 5.6 \\
\hline Other & 7.5 & 7.6 & 9.4 & 5.2 \\
\hline Male, \% & 41.3 & 40.6 & 41.1 & 38.9 \\
\hline Married at Time 2, \% & 47 & 47.1 & 48.8 & 47.6 \\
\hline
\end{tabular}

Column $n$ 's of Early Respondents and Randomized Veterans do not equal 2,100 secondary to exclusions for death, invalid addresses, survey refusal before randomization, and 1 misclassification error.

Bold font signifies a statistically significant difference between early respondents and randomized Veterans.

$* * * p \leq 0.001$ 
Table 2. Sociodemographic Characteristics of Hard to Recruit Survey Recipients by Response Status

\begin{tabular}{lccc}
\hline Characteristic & \multicolumn{2}{c}{$\begin{array}{c}\text { Responded after } \\
\text { Randomization }\end{array}$} & \multirow{2}{*}{ Never Responded } \\
\cline { 2 - 3 } & $\begin{array}{c}\text { Handwritten } \\
n=129\end{array}$ & $\begin{array}{c}\text { Pre-Printed } \\
n=111\end{array}$ & \\
& $56.9(9.2)$ & $55.9(9.8)$ & $54.5(10.6)$ \\
\hline Current age in years, mean (SD) & & & \\
Race \% & 69.5 & 73 & 70.6 \\
$\quad$ White & 16.8 & 18 & 19.2 \\
$\quad$ Black or African American & 6.9 & 6.3 & 6 \\
$\quad$ Hispanic & 11.5 & 5.4 & 6.3 \\
Other & 45.7 & 45 & 36.1 \\
Male\% & 48.1 & 45.9 & 48.9 \\
Married at Time 2, \% & & & \\
\hline
\end{tabular}

